\title{
Ramón de Basterra en Sevilla
}

\author{
Elene Ortega Gallarzagoitia \\ Doctora en Filología Española (U.P.V.) \\ Becaria postdoctoral del Gobierno Vasco
}

Este trabajo aborda el pensamiento histórico de Ramón de Basterra y las posiciones del grupo en el que se integra (la Escuela Romana del Pirineo) en relación con la conferencia que Basterra dicta en Sevilla en mayo de 1921 sobre el emperador Trajano. Se pretende contextualizar en el clasicismo del País Vasco, así como indagar en lo que fue para Basterra el punto de partida de su teoría de la Historia y del conjunto de su obra literaria.

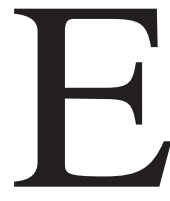

En mayo de 1921 se celebra en Sevilla el Segundo Congreso de Historia y Geografía Hispanoamericanas. Ramón de Basterra (18881928) pronuncia el día 6 en el Ateneo una conferencia titulada «El primer colonizador español, Trajano, y su fundación en Oriente». ${ }^{1}$ Pocos días después repite la conferencia en el Ateneo de Madrid. ${ }^{2}$

El poeta y diplomático bilbaíno acaba de publicar su ensayo La obra de Trajano $^{3}$. Hasta ese momento, Basterra sólo se había dado a conocer por sus colaboraciones en prensa local como Euzkadi, El Nervión, El Pueblo Vasco y El Liberal, además de algunos artículos y poemas en la afamada revista Hermes. Desde su primera juventud el autor vive largos períodos fuera de Bilbao, primero debido a sus estudios, después a su trabajo como diplomático. Sin embargo, ésto no le impide integrase de lleno en la vida cultural del Bilbao de aquellos años y participar en la llamada Escuela Romana del Pirineo.

\footnotetext{
Así lo deduce Guillermo Díaz Plaja en función de la reseña de un artículo del Noticiero Sevillano que publica un periódico bilbaíno: «Una conferencia de Ramón de Bsterra en el Ateneo de Sevilla», El Pueblo Vasco, 8.5.1921 (DIAZ PLAJA, Guillermo: La poesía y el pensamiento de Ramón de Basterra, Juventud, Barcelona, 1941, p. 217)

2 «El primer colonizador español. Trajano y su fundación en Oriente. Conferencia de D. Ramón de Basterra», El Sol, 20.5.1921.

3 BASTERRA, Ramón de: La obra de Trajano, Espasa Calpe, Madrid, 1921.
} 


\section{La Escuela Romana del Pirineo}

En contraste con la pomposidad del nombre, se trata de un grupo informal que no va más allá del voluntarismo del propio Ramón de Basterra y las tertulias vespertinas del café Lyon D'Or en torno a Pedro Eguillor. Pedro Mourlane Michelena, Ramón de Basterra, Fernando de la Quadra Salcedo, José Félix de Lequerica, Rafael Sánchez Mazas y Joaquín de Zuazagotia forman el núcleo del grupo. Estos autores pertenecen a la misma generación y muestran grandes concomitancias ideológicas y estéticas. Comparten un clasicismo que enlaza con Eugenio D’Ors, la Escuela Romana de París, T. E. Hulme, Erza Pound y T. S. Eliot. El grupo bilbaíno posee a un tiempo rasgos peculiares y otros relacionados con la corriente clasicista presente en el resto de España y en Europa:

- Un conservadurismo de talante liberal que evoluciona hacia posturas autoritarias durante los últimos años veinte y los treinta.

- El rechazo manifiesto hacia los nacionalistas vascos y, por supuesto, hacia su visión etnocentrista de la cultura. Basterra y Quadra Salcedo valoran en su juventud aspectos puntuales del bizkaitarrismo, pero no por eso su españolismo es menos evidente que el de otros miembros del grupo.

- La influencia de autores como Sorel, Barres y Maurras. Zuazagoita basa su teoría del «mito coordinador» en las ideas de Sorel. Lequerica se doctora con una tesis sobre este autor y en Sánchez Mazas la influencia de Barres y Maurras aparece en sus escritos sobre todo a partir de los años treinta. ${ }^{4}$ Mourlane manifiesta en sus artículos el interés por los dos neotradicionalistas franceses y es el primer divulgador de Acción Francesa en Bilbao. 5

- el convencimiento de la función socio-política de la élite intelectual y su interés por mantenerse en primera línea de la vida política y cultural de Bilbao, inmersos en las polémicas del momento. Estos autores centran gran parte de su producción literaria en el periódico porque el artículo les permite un posicionamiento inmediato. La Escuela Romana del Pirineo valora la cultura de élite que ellos representan al modo de los ilustrados de la Real Sociedad Vascongada de Amigos del País. ${ }^{6}$

\footnotetext{
Sobre este particular vid. OLABARRIA, Juan: «Para una historia ideológica de la derecha vasca», en Cuadernos de Alzate, $\mathrm{n}^{\circ}$ 15, octubre 1991, pp. 62-53.

5 AREILZA, José María: «El Bilbao de Pedro Eguillor» en VVAA, La Ría que vale millones, Editora Bilbaína, Bilbao, 1957, p. 46

6 MOURLANE MICHELENA, Pedro: «Comentario. Los Amigos del País», Hermes, revista del País Vasco, Edición facsímil, Idatz Ekintza, Bilbao, 1988, Tomo I, nº 1, 1917, p. 23
} 
- Inclinación por la estética clasicista e italianizante. Para este grupo, la cultura de la élite es clasicista. El ensalzamiento de los modelos latinos es un distintivo de cohesión política (frente al nacionalismo vasco) y estética (frente a siglo XIX y el fin de siglo). Sin embargo, esto no impide a Mourlane mostrar su simpatía por representantes del decadentismo finisecular como Huysmans ${ }^{7}$.

- Recuperación de la figura de Peñaflorida y de la labor de la Real Sociedad Vascongada de Amigos del País, considerada la más notable realización del neoclasicismo dieciochesco en el País Vasco. Este tema se refleja en las conferencias y artículos del grupo. Pero es Basterra quien pone mayor empeño con la publicación de una obra que reivindica la influencia de la Real Sociedad Vascongada de Amigos del País en el Nuevo Mundo. ${ }^{8}$

- Antirromanticismo más o menos beligerante, según los casos. El desprecio más acusado hacia la estética del ochocientos está en la pluma de Basterra, mientras que Mourlane tiende a ironizar sobre las formas estéticas que considera caducas. En todo caso, la ostentación clasicista de los miembros de este grupo es un rechazo implícito del romanticismo.

- Confianza en el resurgir artístico de Bilbao, parejo a la prosperidad económica. Dicho resurgir toma un referente tan reiterado como pretencioso: Venecia y Florencia durante el Renacimiento. Mourlane y Sánchez Mazas son quienes más insisten en este paralelismo.

\section{La Teoría de la Historia de Basterra}

En este marco, Basterra desarrolla su peculiar visión de la historia y la cultura. Para la formación de su ideario es fundamental su estancia en Roma como agregado diplomático entre 1915 y 1917. El poeta ha logrado el segundo puesto en las oposiciones y sorprende a sus conocidos cuando elige un destino como Roma. Pero esta elección está motivada por las inquietudes clasicistas del autor. A partir de ese momento sus escritos están impregnados del ideal culturalista que representa el Imperio Romano.

\footnotetext{
«Conferencia de Mourlane», El Liberal, 9.2.1919. La admiración hacia el poeta simbolista Verlaine es patente en el opúsculo de MOURLANE, Pedro: Nuestros amigos los poetas, Bilbao, 1912. Por otra parte, cuando Mourlane dirige La Noche, aparece en este periódico un artículo de Enrique Díez Canedo que ofrece una visión favorable de Verlaine coincidente con la suya (DIEZ CANEDO, Enrique: «De Verlaine, considerado bajo el aspecto de buena persona», La Noche, 10.4.1924)

8 BASTERRA, Ramón de: Una empresa del siglo XVIII. Los Navíos de la Ilustración, Imp. Bolívar, Caracas, 1925 (Reedición en Cultura Hispánica, Madrid, 1970)
} 
Basterra es aficionado a establecer polaridades. Así, en su análisis de la historia, existe Roma y lo que no es Roma, es decir, lo Provincial o Escita. El mundo antiguo está dividido entre los que reciben la luz salvadora de Roma y los que están sumidos en el caos. La misión de la Cuidad es extender la Cultura, imponer el orden, unificar una Europa disgregada. Para Basterra, como para D'Ors, la Historia y la Cultura surgen al mismo tiempo, y sólo son posibles en el ámbito urbano. Este implica una convivencia ordenada, el establecimiento de leyes racionales, el consenso, la armonía, las formas y normas perdurables. El Imperio romano exporta estos valores a toda Europa. Roma introduce a Europa en la Historia.

Basterra proviene del País Vasco, el área hispánica con una romanización más tardía. El poeta se ve a sí mismo como un «bárbaro redento» 9 . Su tierra natal equivale a la Naturaleza, lo espontáneo. Por contra, Roma simboliza la Cultura y el ordenamiento racional, es motor histórico de Europa. Para Basterra, Natura es un ámbito gregario de masas, mientras que Historia «no es sino urdimbre de dirección, mímica y expresión de minoría» 10 .

Es en Roma donde Basterra experimenta el interés hacia el emperador Trajano y el confín oriental del Imperio. La columna de Trajano se le presenta como una señal del camino a seguir:

Fue debajo de la renombrada columna donde me tentó la aventura de ir a la tierra que reflejó Trajano en sus pupilas pías. Del alto monumento caía una larga sombra que asemejaba un índice apuntando la dirección del Oriente. ${ }^{11}$

Así, en junio de 1918 parte hacia Rumanía como miembro de la Legación de España en el país. Transcurre la I Guerra Mundial. Con la ocupación alemana de Bucarest, los diplomáticos se refugian en Jasy. El poeta se enfrenta a graves penurias de las que deja constancia en La obra de Trajano. La ilustración de la portada muestra a la columna trajana erguida sobre un mapa de Rumanía en el que proyecta su sombra. Queda así subrayado el simbolismo que posee para el autor el monumento del Foro romano.

La obra de Trajano es un conjunto de impresiones del Basterra viajero por Rumanía, alternadas con evocaciones históricas que parten de los tiem-

\footnotetext{
9 BASTERRA, Ramón de: Las ubres luminosas, Miguel de Maeztu Editor, Madrid-Bilbao, 1923, p. 37.

${ }^{10}$ BASTERRA, Ramón de: La obra..., op. cit., p. 97

${ }^{11}$ BASTERRA, Ramón de: «Trajano y su obra», en DIAZ PLAJA, Guillermo (ed.): Papeles inéditos y disperos de Ramón de Basterra, Ministerio de Asuntos Exteriores, Madrid, 1970, p. 96.
} 
pos del emperador y llegan hasta el siglo XX. Se suceden repetidamente presente y pasado con la intención de ensalzar la misión civilizadora de Trajano en el la Dacia rebelde.

Bajo las ideas clascistas de Basterra subyace un afán de Unidad que recorre toda su obra. Trajano es el emperador procedente de Hispania -y por tanto del confín occidental del Imperio- que somente y civiliza el extremo oriental. Los habitantes de aquellas tierras son los «escitas», término que Basterra extiende a cualquier «bárbaro» enemigo del Imperio Romano: los pobladores de la Vasconia insurrecta de los tiempos de Augusto son también «escitas», y el ámbito rural vasco contemporáneo al poeta es la «dulce Escitia vascuence». ${ }^{12}$ Los dos extremos rebeldes del Imperio se someten a la Unidad clásica una vez que ha prendido en ellos la «llama romance».

\section{La obra de Basterra}

Con la publicación de La obra de Trajano se abren para Basterra años de gran efervescencia literaria. Además de su intensa actividad como conferenciante en Sevilla, Madrid, Santander, Bilbao y San Sebastián, publica en pocos años tres de sus poemarios: Las ubres luminosas, La sencillez de los seres y Vírulo. Poema de las mocedades. ${ }^{13}$ En 1924 solicita destino en la Embajada Española en Venezuela. Allí escribe y publica su segundo libro en prosa Una empresa del siglo XVIII. Los navíos de la Ilustración. Basterra padece desde hace años una enfermedad mental. A consecuencia de una grave crisis es repatriado y más tarde reanuda sus trabajos diplomáticos en Madrid. Sus dos últimos poemarios ven la luz en 1926: Los labos del monte y Vírulo. Mediodía. ${ }^{14}$

En la mayoría de sus libros, Basterra incluye una lista de obras «del mismo autor» en preparación. El cotejo de estas listas con borradores inéditos confirma que Basterra ambicionaba publicar mucho más. Hacia 1924 el poeta tenía en el cajón o en proyecto, más o menos inacabadas, obras de todo tipo: ensayos y escritos históricos (Dominio Universal de España, Pirineo pensativo), poesía (Llama romance) y teatro (Las alas de lino, Fátima y Las boinas rojas). Incluso comenzó a gestar una tercera parte de Virulo. ${ }^{15}$ Sólo

\footnotetext{
12 Vid. el poema XXIX. «La flota de cachemarines» en BASTERRA, Ramón de: Las ubres luminosas, op. cit.

${ }^{13}$ BASTERRA, Ramón de: La sencillez de los seres, Renacimiento, Madrid, 1923; BASTERRA, Ramón de: Vírulo. Poema de las mocedades, Renacimiento, Madrid, 1924.

${ }^{14}$ BASTERRA, Ramón de: Los labios del monte, Renacimiento, Madrid, 1926; BASTERRA, Ramón de: Vírulo. Mediodía, La Gaceta Literaria, Madrid, 1926.

15 Archivo de la Diputación Foral de Vizcaya.
} 
dos de estas obras vieron la luz de forma póstuma. Guillermo Díaz Plaja editó el poemario inacabado Llama romance y la obra de teatro Las alas de lino ${ }^{16}$

Toda su obra está relacionada con los dos aspectos que marcan sus vida: la tierra natal y los viajes. Así, a cada destino diplomático fuera de España corresponde la gestación de una obra: a Roma Las ubres luminosas, a Rumanía La obra de Trajano y a Caracas Los navios de la Ilustración. Dos poemarios se inspiran en el ámbito natal vasco y pirenaico: La sencillez de los seres y Los labios del monte. El protagonista de Vírulo. Poema de las mocedades -etopeya del propio autor- es oriundo de la misma tierra que Basterra y halla en Madrid la clave de su incipiente ideario. En cuanto a Vírulo. Mediodía, el desarrollo de las tesis del protagonista le permite la integración de todos los ámbitos anteriores.

\section{La conferencia de Sevilla}

Al inicio de su conferencia, Basterra reinvindica la actualidad de la figura de Trajano por dos razones. En primer lugar, por la escasa atención que se presta al emperador Romano en el Congreso de Historia y Geografía Hispanoamericanas donde él mismo participa. La atención se dirige a Hernán Cortés y demás héroes colonizadores del Nuevo Mundo, olvidando que

en la primera forma de civilización que adquirió España, cuando era provincia del Imperio romano, existe la más grande y encantadora figura del colonizador español que es la de Trajano. ${ }^{17}$

Basterra no desdeña a los colonizadores de América. Bien al contrario, considera que España desempeña con la colonización del Nuevo Mundo una labor civilizadora semejante a la que Roma realiza en Europa. Sin embargo, el autor atribuye a Trajano una cualidad superior:

Hernán Cortés, Almagro, Pizarro, héroes fogosos, son arrebato y disparos de energía impremeditada. Trajano es el equilibrio; es el acuerdo de la inteligencia y del brío, del coraje y del pensamiento; teniendo dentro un ideal universal, lo servía con sus fibras, que absorbieron moderación de vuestro clima y de vuestra sangre. ${ }^{18}$

\footnotetext{
16 BASTERRA, Ramón de: Llama romance, Diputación de Vizcaya, Bilbao, 1971; BASTERRA, Ramón de: Las alas de lino, Editora Nacional, Madrid, 1941

17 BASTERRA, Ramón de: «Trajano y su obra», en DIAZ PLAJA, Guillermo (ed.): Papeles inéditos..., op. cit., p. 94

18 op. cit., p. 106
} 
Como se advierte en la última frase de la cita anterior, Basterra atribuye cualidades esenciales de Trajano a un sustrato andaluz, que en último término es un sustrato español: «Trajano es un español del que nos apartan mil años», afirma. Y añade:

A quien dude del españolismo andaluz de Trajano le cito para que concurra al Museo del Capitolio, en Roma, y contemple su estatua. El rostro de Trajano se destaca junto al de los italianos: su aspecto físico es el del español castizo: con un chambergo pavero parecería un picador; con una montera, un espada; de todas suertes, se parece bastante a Lagartijo. ${ }^{19}$

Además del aspecto físico, hay dos cualidades de Trajano que Basterra atribuye «al clima y a la sangre» andaluzas: la delicadeza y el culto a la madre, «tan español, pero especialmente sevillano». ${ }^{20}$

Este emperador es para Basterra savia nueva en la corrompida Roma. En La obra de Trajano, el autor se extiende más en la descripción de unas cualidades tópicas del «princeps», el gran hombre que se sitúa en la cúspide de la élite mandataria: afabilidad, sobriedad, modestia, amor por la disciplina, dotes de estadista, clemencia, dotes militares, camaradería con sus soldados, etc. ${ }^{21}$ Para ello se basa en el Panejírico de Plinio, una fuente que, como se ha descrito recientemente, es interesada y distorsionada. ${ }^{22}$

La visión de Roma que ofrece Basterra -e incluso la de algunos autores que han tratado este tema fundamental de la obra basterriana ${ }^{23}$ - adolece de reduccionismo. La Roma que ofrece el clasicismo contemporáneo es la imagen de Roma construida desde la Antiguiedad por los apologetas imperiales, impulsada de nuevo por la tradición occidental desde el Renacimiento. Interesa sobre todo la Roma Imperial y se deja de lado la Roma republicana, al igual que se ensalzan unos emperadores y se olvidan otros. ${ }^{24}$

Según Basterra, el aporte regenerador de la sangre española en el Imperio debilitado no termina con Trajano. Un contemporáneo suyo, el filósofo Séneca, constituye la otra vertiente de ese caudal salvador para Roma:

\footnotetext{
19 op. cit., p. 94

20 Ibid.

${ }^{21}$ DUPLA, Antonio: «El clasicismo en el País Vasco: Ramón de Basterra», en Vasconia, no 24, 1996, pp. 87.

${ }^{22}$ HIDALGO DE LA VEGA, María José: El intelectual, la realeza y el poder político en el Imperio Romano, Universidad de Salamanca, Salamanca, 1995 (citado por DUPLA, Antonio: op. cit., p. 90)

${ }^{23}$ DIAZ PLAJA, Guillermo: La poesía y el pensamiento..., op. cit.; AREAN, Carlos Antonio: Ramón de Basterra, Cultura Hispánica, Madrid, 1953; ELIZALDE, Ignacio: «Ramón de Basterra y el mundo clásico», en Letras de Deusto, no 27, diciembre 1983, pp. 47-66.

${ }^{24}$ DUPLA, Antonio: op. cit., p. 89.
} 
Pocos años antes de la marcha de Trajano a la Eterna Ciudad se había trasladado allí desde Córdoba el padre de Séneca con sus tres hijos. Séneca y Trajano representan la entrada en acción de España en los destinos de Roma. Cuando la sangre italiana testimoniaba cansancio, del Occidente español llegó el renuevo de entereza que en las letras y las armas obtuvo el decadente esfuerzo, a pique de ruina. ${ }^{25}$

Esta alusión a Trajano y a Séneca como adalides respectivos de las armas y las letras trae a la memoria un texto señalado entre la producción de los miembros de la Escuela Romana del Pirineo: El discurso de las armas y las letras de Pedro Mourlane Michelena ${ }^{26}$. En él no se habla de ninguno de los dos personajes clásicos, pero el clasicismo condiciona la visión de la I Guerra Mundial. Este es el acontecimiento que motiva y centra la obra. La aliadofilia de Mourlane se puede inscribir en el prolatinismo que comparten muchos aliadófilos del momento ${ }^{27}$. En El discurso de las armas y las letras la guerra tiene un valor estético e ideológico relacionado con el ideal clasicista presente en toda la obra. La epopeya imperial romana es un referente implícito que a veces se manifiesta con claridad. Así sucede cuando compara al militar francés Joffre con el dictador romano del siglo III a. C. Quinto Fabio. Tal como anuncia el título de la obra, el ideal de Mourlane (común a los escritores de su grupo) es la conjunción de armas y letras, de acción y pensamiento. En último término, Roma constituye un paradigma de ese ideal, ya que su imperialismo fue tanto bélico como cultural.

Basterra habla de las victorias bélicas de Trajano en la Dacia rebelde, pero no olvida la pervivencia del sustrato latino en la lengua y en la lírica de aquellas tierras. El autor se complace en encontrar similitudes entre palabras, canciones populares y romances de España y de Rumanía. El recorrido por la historia rumana desde la conquista de Trajano hasta el siglo XX tiene la finalidad de mostrar cómo las sucesivas invasiones (húngaros, eslavos, turcos...) y los avatares históricos han relegado el sustrato latino al campesinado:

25 BASTERRA, Ramón de: «Trajano y su obra», en DIAZ PLAJA, Guillermo (ed.): Papeles inéditos..., op. cit., p. 95

${ }^{26}$ MOURLANE MICHELENA, Pedro: El discurso de las armas y las letras, Biblioteca de Amigos del País, Bilbao, 1915.

27 Vid. DIAZ PLAJA, Fernando: Francófilos y germanófilos. Los españoles ante la guerra europea, Dopesa, Barcelona, 1972 y LITVAK, Lily: «Latinos y anglosajones. Una polémica de la España de fin de siglo», en España 1900. Anarquismo, modernismo y fin de siglo, Anthropos, Barcelona, 1990. 
Los acontecimientos dispusieron que este pueblo fuera por miles de años el campesino perpetuo. La aristocracia de propietarios de tierra levantada de su seno, que fue en el pasado su amparo y guía, perdió bastantes de las tradiciones afincadas en el suelo, conversando en sus estancias pulidas, aquí en húngaro y alemán, allí en griego, o en francés más tarde; más lejos en ruso. Fueron, en consecuencia, el montañés y el labriego quienes conservaron sus canciones, trajes y usos, las reliquias del pasado. En el fondo Rumanía es una perduración, en formas aldeanas, de la viejísima fundación trajana. ${ }^{28}$

Las últimas oleadas de invasores se han producido durante la Gran Guerra: los ejércitos imperiales alemanes, austro-húngaros, turcos y búlgaros.

Llegamos así al segundo de los motivos por el cual Basterra defiende la importancia y la actualidad del emperador Trajano. El mismo ha sido testigo, durante su estancia como diplomático en Rumanía, de los acontecimientos que culminan en la unificación de Rumanía tras la I Guerra Mundial. Tanto en La obra de Trajano como en la conferencia describe el fervor con el que el pueblo rumano evoca al que considera su fundador y artífice primero de su unidad: Trajano. El poeta narra el modo en que los diplomáticos españoles son aclamados por la población cuando por fin Rumanía se ve unificada y libre de invasores:

Aguardaban numerosos grupos de estudiantes; pasaron ante ellos, que los dejaron transcurrir en silencio, los poderosos representantes de los Estados Unidos, de Inglaterra, de Francia y de Italia; sólo al divisar a la representación de nuestra Patria prorrumpieron los grupos en entusiastas gritos de ¡Viva España!.29

Basterra considera a Sevilla, como a ningún otro lugar de España, la depositaria de este fervor nacido en el que fue el extremo oriental del Imperio romano. $\mathrm{Y}$ aunque reconoce que existe una «Calle de Trajano» en Sevilla, evoca por comparación una nación, encima de la península de los Balcanes, cuyo nombre es Rumanía, que apenas cuenta con una ciudad ni casi con una aldea que no luzca en una plaza, hotel o anuncio, el nombre de Trajano. ${ }^{30}$

\footnotetext{
${ }^{28}$ BASTERRA, Ramón de: «Trajano y su obra», en DIAZ PLAJA, Guillermo (ed.): Papeles inéditos..., op. cit., p. 104.

29 op. cit., p. 105

${ }^{30}$ op. cit., p. 96
} 
Con su disertación en Sevilla, Basterra pretende reavivar la figura de Trajano entre los andaluces. A ellos manifiesta, con su vehemencia habitual, las «gracias rendidas por haberle dado nacimiento». 31

Termina de este modo una conferencia que sorprende al lector de hoy día por la visión maniquea de la historia rumana y romana. Basterra divide a los invasores de Rumanía en buenos y malos, civilizadores y bárbaros. Justifica la crudeza de la invasión trajana, y critica el resto de las oleadas. Es evidente que Basterra simplifica la historia. Pero a la luz de su obra en conjunto, se advierte que el poeta creía con pasión en las ideas históricas sobre las que escribe. Tanto es así, que en algunos momentos consigue transmitir su emoción ante hechos históricos pasados y presentes, y su cariño por unas «bellas y tristes regiones» que forman el que fue «uno de los países más desventurados de la Tierra ${ }^{32}$. La causa de esa desventura no es otra que el haber sido alejado durante siglos de sus raíces clásicas. 


\section{Bibliografía}

ANONIMO: «Conferencia de Mourlane», El Liberal, 9.2.1919.

- «Una conferencia de Ramón de Basterra en el Ateneo de Sevilla», El Pueblo Vasco, 8.5.1921

- «El primer colonizador español. Trajano y su fundación en Oriente. Conferencia de D. Ramón de Basterra», El Sol, 20.5.1921.

AREAN, Carlos Antonio: Ramón de Basterra, Cultura Hispánica, Madrid, 1953.

AREILZA, José María: «El Bilbao de Pedro Eguillor» en VVAA, La Ría que vale millones, Editora Bilbaína, Bilbao, 1957.

BASTERRA, Ramón de: La obra de Trajano, Espasa Calpe, Madrid, 1921.

- Las ubres luminosas, Miguel de Maeztu Editor, Madrid-Bilbao, 1923

- La sencillez de los seres, Renacimiento, Madrid, 1923

- Vírulo. Poema de las mocedades, Renacimiento, Madrid, 1924.

- Una empresa del siglo XVIII. Los Navíos de la Ilustración, Imp. Bolívar, Caracas, 1925 (Reedición en Cultura Hispánica, Madrid, 1970).

- Los labios del monte, Renacimiento, Madrid, 1926

- Vírulo. Mediodía, La Gaceta Literaria, Madrid, 1926.

- Las alas de lino, Editora Nacional, Madrid, 1941

- Llama romance, Diputación de Vizcaya, Bilbao, 1971

DIAZ PLAJA, Fernando: Francófilos y germanófilos. Los españoles ante la guerra europea, Dopesa, Barcelona, 1972 .

DIAZ PLAJA, Guillermo: La poesía y el pensamiento de Ramón de Basterra, Juventud, Barcelona, 1941.

- (ed.): Papeles inéditos y disperos de Ramón de Basterra, Ministerio de Asuntos Exteriores, Madrid, 1970, p. 96.

DIEZ CANEDO, Enrique: «De Verlaine, considerado bajo el aspecto de buena persona», La Noche, 10.4.1924.

DUPLA, Antonio: «El clasicismo en el País Vasco: Ramón de Basterra», en Vasconia, no 24, 1996, pp. 81-100.

ELIZALDE, Ignacio: «Ramón de Basterra y el mundo clásico», en Letras de Deusto, $\mathrm{n}^{\circ}$ 27, diciembre 1983, pp. 47-66.

LITVAK, Lily: España 1900. Anarquismo, modernismo y fin de siglo, Anthropos, Barcelona, 1990.

MOURLANE MICHELENA, Pedro: Nuestros amigos los poetas, Bilbao, 1912. 
- El discurso de las armas y las letras, Biblioteca de Amigos del País, Bilbao, 1915.

- «Comentario. Los Amigos del País», Hermes, revista del País Vasco, Edición facsímil, Idatz Ekintza, Bilbao, 1988, Tomo I, nº 1, 1917, pp. 2123.

OLABARRIA, Juan: «Para una historia ideológica de la derecha vasca», en Cuadernos de Alzate, $\mathrm{n}^{\circ}$ 15, octubre 1991, pp. 62-53. 